\title{
On the Stability of Solutions of Nonlinear Functional Differential Equation of the Fifth-Order
}

\author{
A. M. A. Abou-El-Ela1', A. I. Sadek' ${ }^{\text {, A. M. Mahmoud }}{ }^{2}$, R. O. A. Taie ${ }^{1}$ \\ ${ }^{1}$ Department of Mathematics, Faculty of Science, Assiut University, Assiut, Egypt \\ ${ }^{2}$ Department of Mathematics, Faculty of Science, New Valley Branch, Assiut University, New Valley, El-Khargah, \\ Egypt \\ Email: a.el-ela@aun.edu.eg, Sadeka1961@hotmail.com, math ayman27@yahoo.com, \\ Rasha omath@yahoo.com
}

Received 17 June 2014; revised 15 July 2014; accepted 21 July 2014

Copyright $@ 2014$ by authors and Scientific Research Publishing Inc.

This work is licensed under the Creative Commons Attribution International License (CC BY).

http://creativecommons.org/licenses/by/4.0/

(c) () Open Access

\section{Abstract}

The main purpose of this paper is to investigate global asymptotic stability of the zero solution of the fifth-order nonlinear delay differential equation on the following form

$$
\begin{aligned}
& x^{(5)}(t)+\psi\left(x(t-r), \dot{x}(t-r), \ddot{x}(t-r), \dddot{x}(t-r), x^{(4)}(t-r)\right) x^{(4)}(t) \\
& +f(\dddot{x}(t-r))+\phi(\ddot{x}(t-r))+g(\dot{x}(t))+h(x(t))=0 .
\end{aligned}
$$

By constructing a Lyapunov functional, sufficient conditions for the stability of the zero solution of this equation are established.

\section{Keywords}

Global Asymptotic Stability, Lyapunov Functional, Fifth-Order Delay Differential Equations

\section{Introduction}

As is well-known, the area of differential equations is an old but durable subject, that remains alive and useful to a wide variety of engineers, scientists and mathematicians. Now the subject of differential equations represents a huge body of knowledge including many subfields and a vast array of applications in many disciplines. It should be noted that principles of differential equations are largely related to the qualitative theory of ordinary differential equations. Qualitative theory refers to the study of behaviour of solutions, for example, the investigation of 
stability, instability, boundedness of solutions and etc., without determining explicit formulas for the solutions. In particular one can refer that many authors have dealt with delay differential equations and its problems, and many excellent results have been obtained on the behaviour of solutions for various higher-order: second-, third-, fourth-, and fifth-order nonlinear differential equations with delay, for example, [1]-[27], and references quoted therein, which contain the differential equations without delay or with delay. In many of these references, the authors dealt with the problems by using Lyapunov's second method [28]. By considering Lyapunov functionals we obtained the conditions which ensured the stability of the problem. It is worth-mentioning that construction of these Lyapunov functionals remains a general problem. We know that a similar problem exists for ordinary differential equations for higher-order [12]. Clearly, it is even more difficult to construct Lyapunov functionals for delay differential equations of higher-order. Up to this moment the investigations concerning the stability of solutions of nonlinear equations of fifth-order with delay have not been fully developed.

In particular in 2010 Tunç [29] obtained sufficient conditions, which ensure the stability of the zero solution of a nonlinear delay differential equation of fifth-order

$$
\begin{aligned}
& x^{(5)}(t)+\psi\left(x(t-r), \dot{x}(t-r), \ddot{x}(t-r), \dddot{x}(t-r), x^{(4)}(t-r)\right) x^{(4)}(t) \\
& +f(\ddot{x}(t-r), \dddot{x}(t-r))+\alpha_{3} \ddot{x}(t)+\alpha_{4} \dot{x}(t)+\alpha_{5} x(t)=0,
\end{aligned}
$$

where $\psi$ and $f$ are continuous functions; $\alpha_{3}, \alpha_{4}$ and $\alpha_{5}$ are positive constants, $r$ is a bounded delay and positive constant; the derivatives $f_{z}(z, w), f_{w}(z, w)$ exist and are continuous for all $z, w$ and $f(z, 0)=0$.

Later in 2011 Abou-El-Ela, Sadek and Mahmoud [30] obtained the sufficient conditions for the uniform stability of the zero solution of a nonlinear fifth-order delay differential equation of the following form

$$
x^{(5)}(t)+\phi(\dddot{x}(t)) x^{4}(t)+\psi(\dddot{x}(t))+f(\ddot{x}(t))+g(\dot{x}(t))+h(x(t-r))=0,
$$

where $r$ is a positive constant; $\phi(\dddot{x}), \psi(\dddot{x}), f(\ddot{x}), g(\dot{x})$ and $h(x)$ are continuous functions and $\psi(0)=f(0)=g(0)=h(0)=0$.

In the present paper, we are concerned with the stability of the zero solution of the fifth-order nonlinear delay differential equation on the form

$$
\begin{aligned}
& x^{(5)}(t)+\psi\left(x(t-r), \dot{x}(t-r), \ddot{x}(t-r), \dddot{x}(t-r), x^{(4)}(t-r)\right) x^{(4)}(t) \\
& +f(\dddot{x}(t-r))+\phi(\ddot{x}(t-r))+g(\dot{x}(t))+h(x(t))=0,
\end{aligned}
$$

or its equivalent system form

$$
\begin{aligned}
\dot{x}= & y, \quad \dot{y}=z, \quad \dot{z}=w, \quad \dot{w}=u, \\
\dot{u}= & -\psi(x(t-r), y(t-r), z(t-r), w(t-r), u(t-r)) u-f(w) \\
& -\phi(z)-g(y)-h(x)+\int_{t-r}^{t} f^{\prime}(w(s)) u(s) \mathrm{d} s+\int_{t-r}^{t} \phi^{\prime}(z(s)) w(s) \mathrm{d} s,
\end{aligned}
$$

where $\psi, f, \phi, g$ and $h$ are continuous functions for the arguments displayed explicitly in (1.1) with $f(0)=\phi(0)=g(0)=h(0)=0, \quad r$ is a bounded delay and positive constant; the derivatives $f^{\prime}(w)$ and $\phi^{\prime}(z)$ exist and are continuous for all $w, z$.

\section{Preliminaries and Stability Results}

In order to reach the main result of this paper, we will give some basic information to the stability criteria for the general autonomous delay differential system. We consider

$$
\dot{x}=f\left(x_{t}\right), x_{t}=x(t+\theta),-r \leq \theta \leq 0, t \geq 0,
$$

where $f: C_{H} \rightarrow \mathbb{R}^{n}$ is a continuous mapping, $f(0)=0, C_{H}:=\left\{\varphi \in C\left([-r, 0], \mathbb{R}^{n}\right):\|\varphi\| \leq H\right\}$ and for $H_{1}<H$, there exists $L\left(H_{1}\right)>0$, with $|f(\varphi)| \leq L\left(H_{1}\right)$ when $\|\varphi\| \leq H_{1}$.

The following are the classical theorems on uniform stability and global asymptotic stability for the solution of (2.1).

Theorem 2.1. [31]. Let $V(\varphi): \mathcal{C}_{H} \rightarrow \mathbb{R}$ be a continuous functional satisfying a local Lipschitz condition $V(0)=0$ and the functions $W_{i}(r),(i=1,2)$ are wedges such that 
i) $W_{1}(|\varphi(0)|) \leq V(\varphi) \leq W_{2}(\|\varphi\|)$ and

ii) $\dot{V}_{(2,1)}(\varphi) \leq 0$.

Then the zero solution of (2.1) is uniformly stable.

Theorem 2.2. [32]. Suppose $f(0)=0$, let $V$ be a continuous functional defined on $C_{H}=C$ with $V(0)=0$, and let $u(s)$ be non-negative and continuous function for $0 \leq s<\infty, u(s) \rightarrow \infty$ as $s \rightarrow \infty$ such that for all $\varphi \in C$

i) $u(|\varphi(0)|) \leq V(\varphi), \quad V(\varphi) \geq 0$ and

ii) $\dot{V}_{(2,1)}(\varphi)<0$ for $\varphi \neq 0$.

Then all solutions of (2.1) approach zero as $t \rightarrow \infty$ and the origin is globally asymptotically stable.

The following will be our main stability result for (1.1).

Theorem 2.3. In addition to the basic assumptions imposed on the functions $\psi, f, \phi, g$ and $h$. Suppose that the following conditions are satisfied, where $\alpha_{1}, \cdots, \alpha_{5}$ are arbitrary positive constants and $\varepsilon, \varepsilon_{o}, \delta, \lambda, \rho, \rho_{1}, M$ and $L$ are sufficiently small positive constants

i) $\alpha_{1}>0, \alpha_{1} \alpha_{2}-\alpha_{3}>0, \alpha_{5}>0,\left(\alpha_{1} \alpha_{2}-\alpha_{3}\right) \alpha_{3}-\left(\alpha_{1} \alpha_{4}-\alpha_{5}\right) \alpha_{1}>0$.

$$
\delta_{o}=\left(\alpha_{3} \alpha_{4}-\alpha_{2} \alpha_{5}\right)\left(\alpha_{1} \alpha_{2}-\alpha_{3}\right)-\left(\alpha_{1} \alpha_{4}-\alpha_{5}\right)^{2}>0,
$$

and the following two inequalities

$$
\begin{gathered}
\Delta_{1}=\frac{\left(\alpha_{3} \alpha_{4}-\alpha_{2} \alpha_{5}\right)\left(\alpha_{1} \alpha_{2}-\alpha_{3}\right)}{\alpha_{1} \alpha_{4}-\alpha_{5}}-\left\{\alpha_{1} g^{\prime}(y)-\alpha_{5}\right\}>2 \epsilon \alpha_{2} \\
\Delta_{2}=\frac{\alpha_{3} \alpha_{4}-\alpha_{2} \alpha_{5}}{\alpha_{1} \alpha_{4}-\alpha_{5}}-\frac{\left(\alpha_{1} \alpha_{4}-\alpha_{5}\right) \gamma(y)}{\alpha_{4}\left(\alpha_{1} \alpha_{2}-\alpha_{3}\right)}-\frac{\epsilon}{\alpha_{1}}>0,
\end{gathered}
$$

for all $y$ and all $t \in \mathbb{R}^{+}$, where

$$
\gamma(y)= \begin{cases}g(y) / y, & y \neq 0 \\ g^{\prime}(0), & y=0\end{cases}
$$

ii) $2 \epsilon_{o} \leq \psi(x(t-r), y(t-r), z(t-r), w(t-r), u(t-r))-\alpha_{1} \leq \min \left\{\frac{\epsilon}{12 \alpha_{1}^{2}} \frac{\epsilon \alpha_{2}\left(\alpha_{1} \alpha_{4}-\alpha_{5}\right)^{2}}{14 \alpha_{4}^{2}\left(\alpha_{1} \alpha_{2}-\alpha_{3}\right)^{2}}, \frac{\epsilon \alpha_{4}}{12 \delta^{2}}\right\}$.

iii) $f(0)=0, \frac{f(w)}{w} \geq \alpha_{2} ; w \neq 0,\left|f^{\prime}(w(s))\right| \leq M \quad$ and

$$
\left[\frac{f(w)}{w}-\alpha_{2}\right]^{2} \leq \min \left\{\frac{2 \varepsilon^{2} \alpha_{2}\left(\alpha_{1} \alpha_{4}-\alpha_{5}\right)^{2}}{21 \alpha_{4}^{2}\left(\alpha_{1} \alpha_{2}-\alpha_{3}\right)^{2}}, \frac{\varepsilon^{2} \alpha_{4}}{9 \delta^{2}}\right\}
$$

iv) $\phi(0)=0, \frac{\phi(z)}{z} \geq \alpha_{3} ; z \neq 0,\left|\phi^{\prime}(z(s))\right| \leq L$ and

$$
\left[\frac{\phi(z)}{z}-\alpha_{3}\right]^{2} \leq \min \left\{\frac{\epsilon \epsilon_{o} \alpha_{2}}{7}, \frac{2 \epsilon^{2} \alpha_{2} \alpha_{4}}{21 \delta^{2}}\right\} .
$$

v) $g(0)=0, \frac{g(y)}{y} \geq \alpha_{4}, g^{\prime}(y)-\frac{g(y)}{y} \leq \frac{\alpha_{5} \delta_{o}}{\alpha_{4}^{2}\left(\alpha_{1} \alpha_{2}-\alpha_{3}\right)} ; y \neq 0$ and

$$
\left[\alpha_{4}-g^{\prime}(y)\right]^{2} \leq \frac{2 \epsilon^{2} \alpha_{2}}{21} \text {. }
$$


vi) $h(0)=0, h(x) \operatorname{sgn} x>0(x \neq 0), H(x) \equiv \int_{0}^{x} h(\xi) \mathrm{d} \xi \rightarrow \infty$ as $|x| \rightarrow \infty, h^{\prime}(x) \leq \alpha_{5}$ for all $x$, and

$$
\left[\alpha_{5}-h^{\prime}(x)\right]^{2} \leq \min \left\{\frac{\epsilon^{2} \alpha_{4}}{9}, \frac{2 \epsilon^{2} \alpha_{2} \alpha_{4}}{21 \delta^{2}}\right\} .
$$

Then the zero solution of (1.1) is globally asymptotically stable, provided that

$$
r<\min \left\{\frac{\epsilon \alpha_{4}}{6 \delta(L+M)}, \frac{\epsilon \alpha_{2}\left(\alpha_{1} \alpha_{4}-\alpha_{5}\right)}{7 \alpha_{4}(L+M)\left(\alpha_{1} \alpha_{2}-\alpha_{3}\right)}, \frac{\epsilon}{6 \alpha_{1}(L+M)+12 \lambda}, \frac{\epsilon_{o}}{L+M+2 \rho}\right\} .
$$

Proof. We define the Lyapunov functional $V=V\left(x_{t}, y_{t}, z_{t}, w_{t}, u_{t}\right)$ as:

$$
\begin{aligned}
2 V\left(x_{t}, y_{t}, z_{t}, w_{t}, u_{t}\right)= & u^{2}+2 \alpha_{1} u w+\frac{2 \alpha_{4}\left(\alpha_{1} \alpha_{2}-\alpha_{3}\right)}{\alpha_{1} \alpha_{4}-\alpha_{5}} u z+2 \delta u y+2 \int_{0}^{w} f(\sigma) \mathrm{d} \sigma \\
& +\left[\alpha_{1}^{2}-\frac{\alpha_{4}\left(\alpha_{1} \alpha_{2}-\alpha_{3}\right)}{\alpha_{1} \alpha_{4}-\alpha_{5}}\right] w^{2}+2\left[\alpha_{3}+\frac{\alpha_{1} \alpha_{4}\left(\alpha_{1} \alpha_{2}-\alpha_{3}\right)}{\alpha_{1} \alpha_{4}-\alpha_{5}}-\delta\right] w z \\
& +2 \alpha_{1} \delta w y+2 w g(y)+2 w h(x)+2 \alpha_{1} \int_{0}^{z} \phi(\zeta) \mathrm{d} \zeta \\
& +\left[\frac{\alpha_{2} \alpha_{4}\left(\alpha_{1} \alpha_{2}-\alpha_{3}\right)}{\alpha_{1} \alpha_{4}-\alpha_{5}}-\alpha_{4}-\alpha_{1} \delta\right] z^{2}+2 \delta \alpha_{2} y z+2 \alpha_{1} z g(y) \\
& -2 \alpha_{5} y z+2 \alpha_{1} z h(x)+\frac{2 \alpha_{4}\left(\alpha_{1} \alpha_{2}-\alpha_{3}\right)}{\alpha_{1} \alpha_{4}-\alpha_{5}} \int_{0}^{y} g(\eta) \mathrm{d} \eta \\
& +\left(\delta \alpha_{3}-\alpha_{1} \alpha_{5}\right) y^{2}+\frac{2 \alpha_{4}\left(\alpha_{1} \alpha_{2}-\alpha_{3}\right)}{\alpha_{1} \alpha_{4}-\alpha_{5}} y h(x)+2 \delta \int_{0}^{x} h(\xi) \mathrm{d} \xi \\
& +2 \lambda \int_{-r}^{0} \int_{t+s}^{t} w^{2}(\theta) \mathrm{d} \theta \mathrm{d} s+2 \rho \int_{-r}^{0} \int_{t+s}^{t} u^{2}(\theta) \mathrm{d} \theta \mathrm{d} s,
\end{aligned}
$$

where $\rho$ and $\lambda$ are two positive constants, which will be determined later and $\delta$ is a positive constant defined by

$$
\delta:=\frac{\alpha_{5}\left(\alpha_{1} \alpha_{2}-\alpha_{3}\right)}{\alpha_{1} \alpha_{4}-\alpha_{5}}+\epsilon .
$$

Then it is convenient to rewrite the expression for the Lyapunov functional defined in (2.5) in the following form

$$
\begin{aligned}
2 V= & \left\{u+\alpha_{1} w+\frac{\alpha_{4}\left(\alpha_{1} \alpha_{2}-\alpha_{3}\right)}{\alpha_{1} \alpha_{4}-\alpha_{5}} z+\delta y\right\}^{2}+\frac{\alpha_{4} \delta_{o}}{\left(\alpha_{1} \alpha_{4}-\alpha_{5}\right)^{2}}\left(z+\frac{\alpha_{5}}{\alpha_{4}} y\right)^{2} \\
& +\frac{\alpha_{4}\left(\alpha_{1} \alpha_{4}-\alpha_{5}\right)}{\left(\alpha_{1} \alpha_{2}-\alpha_{3}\right) \gamma}\left\{\frac{\alpha_{1} \alpha_{2}-\alpha_{3}}{\alpha_{1} \alpha_{4}-\alpha_{5}} h(x)+\frac{\alpha_{1} \alpha_{2}-\alpha_{3}}{\alpha_{1} \alpha_{4}-\alpha_{5}} \gamma y+\frac{\alpha_{1}}{\alpha_{4}} \gamma z+\frac{1}{\alpha_{4}} \gamma w\right\}^{2} \\
& +\Delta_{2}\left(w+\alpha_{1} z\right)^{2}+2 \epsilon\left(\frac{\alpha_{3} \alpha_{4}-\alpha_{2} \alpha_{5}}{\alpha_{1} \alpha_{4}-\alpha_{5}}\right) y z+2 \lambda \int_{-r}^{0} \int_{t+s}^{t} w^{2}(\theta) \mathrm{d} \theta \mathrm{d} s \\
& +2 \rho \int_{-r}^{0} \int_{t+s}^{t} u^{2}(\theta) \mathrm{d} \theta \mathrm{d} s+\sum_{i=1}^{4} v_{i},
\end{aligned}
$$

where

$$
v_{1}:=2 \delta \int_{0}^{x} h(\xi) \mathrm{d} \xi-\frac{\alpha_{4}\left(\alpha_{1} \alpha_{2}-\alpha_{3}\right)}{\left(\alpha_{1} \alpha_{4}-\alpha_{5}\right) \gamma} h^{2}(x)
$$




$$
\begin{gathered}
v_{2}:=\frac{\alpha_{4}\left(\alpha_{1} \alpha_{2}-\alpha_{3}\right)}{\alpha_{1} \alpha_{4}-\alpha_{5}}\left[2 \int_{0}^{y} g(\eta) \mathrm{d} \eta-y g(y)\right]+\left[\delta \alpha_{3}-\alpha_{1} \alpha_{5}-\frac{\alpha_{5}^{2} \delta_{o}}{\alpha_{4}\left(\alpha_{1} \alpha_{4}-\alpha_{5}\right)^{2}}-\delta^{2}\right] y^{2}, \\
v_{3}:=\frac{\epsilon}{\alpha_{1}} w^{2}+2 \int_{0}^{w} f(\sigma) \mathrm{d} \sigma-\alpha_{2} w^{2}, \\
v_{4}:=2 \alpha_{1} \int_{0}^{z} \phi(\zeta) \mathrm{d} \zeta-\alpha_{1} \alpha_{3} z^{2} .
\end{gathered}
$$

For the component $v_{1}$, by using (2.6) and the definition of $\gamma$

$$
\begin{aligned}
v_{1} & =2 \epsilon \int_{0}^{x} h(\xi) \mathrm{d} \xi+\frac{2 \alpha_{5}\left(\alpha_{1} \alpha_{2}-\alpha_{3}\right)}{\alpha_{1} \alpha_{4}-\alpha_{5}} \int_{0}^{x} h(\xi) \mathrm{d} \xi-\frac{\alpha_{4}\left(\alpha_{1} \alpha_{2}-\alpha_{3}\right)}{\left(\alpha_{1} \alpha_{4}-\alpha_{5}\right) \gamma} h^{2}(x) \\
& =2 \epsilon \int_{0}^{x} h(\xi) \mathrm{d} \xi+\frac{\alpha_{1} \alpha_{2}-\alpha_{3}}{\alpha_{1} \alpha_{4}-\alpha_{5}}\left\{2 \alpha_{5} \int_{0}^{x} h(\xi) \mathrm{d} \xi-\frac{\alpha_{4}}{\gamma} h^{2}(x)\right\},
\end{aligned}
$$

since $\frac{\alpha_{4}}{\gamma} \leq 1$ by v), thus we obtain

$$
2 \alpha_{5} \int_{0}^{x} h(\xi) \mathrm{d} \xi-\frac{\alpha_{4}}{\gamma} h^{2}(x)=2 \int_{0}^{x}\left\{\alpha_{5}-h^{\prime}(\xi)\right\} h(\xi) \mathrm{d} \xi-h^{2}(0) \geq 0 .
$$

This is due to the fact that the integral on the right-hand side is non-negative by vi), therefore we get

$$
v_{1} \geq 2 \epsilon \int_{0}^{x} h(\xi) \mathrm{d} \xi
$$

From the identity

$$
y g(y) \equiv \int_{0}^{y} g(\eta) \mathrm{d} \eta+\int_{0}^{y} \eta g^{\prime}(\eta) \mathrm{d} \eta,
$$

therefore

$$
v_{2} \geq \int_{0}^{y}\left[\frac{\alpha_{5} \delta_{o}}{\alpha_{4}\left(\alpha_{1} \alpha_{4}-\alpha_{5}\right)}-2 \epsilon\left\{\epsilon+\frac{2 \alpha_{5}\left(\alpha_{1} \alpha_{2}-\alpha_{3}\right)}{\alpha_{1} \alpha_{4}-\alpha_{5}}-\alpha_{3}\right\}\right] \eta \mathrm{d} \eta \geq \frac{\alpha_{5} \delta_{o}}{4 \alpha_{4}\left(\alpha_{1} \alpha_{4}-\alpha_{5}\right)} y^{2},
$$

and by using v) we find

$$
v_{2} \geq \int_{0}^{y}\left[\frac{\alpha_{5} \delta_{o}}{\alpha_{4}\left(\alpha_{1} \alpha_{4}-\alpha_{5}\right)}-2 \epsilon\left\{\epsilon+\frac{2 \alpha_{5}\left(\alpha_{1} \alpha_{2}-\alpha_{3}\right)}{\alpha_{1} \alpha_{4}-\alpha_{5}}-\alpha_{3}\right\}\right] \eta \mathrm{d} \eta \geq \frac{\alpha_{5} \delta_{o}}{4 \alpha_{4}\left(\alpha_{1} \alpha_{4}-\alpha_{5}\right)} y^{2},
$$

provided that

$$
\begin{gathered}
\frac{\alpha_{5} \delta_{o}}{4 \alpha_{4}\left(\alpha_{1} \alpha_{4}-\alpha_{5}\right)} \geq \epsilon\left\{\epsilon+\frac{2 \alpha_{5}\left(\alpha_{1} \alpha_{2}-\alpha_{3}\right)}{\alpha_{1} \alpha_{4}-\alpha_{5}}-\alpha_{3}\right\} . \\
v_{3}=\frac{\epsilon}{\alpha_{1}} w^{2}+2 \int_{0}^{w}\left\{\frac{f(\sigma)}{\sigma}-\alpha_{2}\right\} \sigma \mathrm{d} \sigma \geq \frac{\epsilon}{\alpha_{1}} w^{2}, \text { by (iii). }
\end{gathered}
$$

From iv) we find

$$
v_{4}=2 \alpha_{1} \int_{0}^{z}\left\{\frac{\phi(\zeta)}{\zeta}-\alpha_{3}\right\} \zeta \mathrm{d} \zeta \geq 0
$$


Summing up the four inequalities obtained from $v_{1}, \cdots, v_{4}$ into (2.7), we have

$$
\begin{aligned}
2 V \geq & \left\{u+\alpha_{1} w+\frac{\alpha_{4}\left(\alpha_{1} \alpha_{2}-\alpha_{3}\right)}{\alpha_{1} \alpha_{4}-\alpha_{5}} z+\delta y\right\}^{2}+\frac{\alpha_{4} \delta_{o}}{\left(\alpha_{1} \alpha_{4}-\alpha_{5}\right)^{2}}\left(z+\frac{\alpha_{5}}{\alpha_{4}} y\right)^{2} \\
& +\Delta_{2}\left(w+\alpha_{1} z\right)^{2}+2 \epsilon \int_{0}^{x} h(\xi) \mathrm{d} \xi+\frac{\alpha_{5} \delta_{o}}{4 \alpha_{4}\left(\alpha_{1} \alpha_{4}-\alpha_{5}\right)} y^{2}+\frac{\epsilon}{\alpha_{1}} w^{2} \\
& +2 \epsilon\left(\frac{\alpha_{3} \alpha_{4}-\alpha_{2} \alpha_{5}}{\alpha_{1} \alpha_{4}-\alpha_{5}}\right) y z+2 \lambda \int_{-r}^{0} \int_{t+s}^{t} w^{2}(\theta) \mathrm{d} \theta \mathrm{d} s+2 \rho \int_{-r}^{0} \int_{t+s}^{t} u^{2}(\theta) \mathrm{d} \theta \mathrm{d} s .
\end{aligned}
$$

Clearly, it follows from the first six terms included in (2.8) that there exist sufficiently small positive constants $D_{i},(i=1, \cdots, 5)$, such that

$$
\begin{aligned}
2 V \geq & D_{1} H(x)+2 D_{2} y^{2}+2 D_{3} z^{2}+D_{4} w^{2}+D_{5} u^{2}+2 \epsilon\left(\frac{\alpha_{3} \alpha_{4}-\alpha_{2} \alpha_{5}}{\alpha_{1} \alpha_{4}-\alpha_{5}}\right) y z \\
& +2 \lambda \int_{-r}^{0} \int_{t+s}^{t} w^{2}(\theta) \mathrm{d} \theta \mathrm{d} s+2 \rho \int_{-r}^{0} \int_{t+s}^{t} u^{2}(\theta) \mathrm{d} \theta \mathrm{d} s .
\end{aligned}
$$

Now we consider the terms

$$
v_{5}:=D_{2} y^{2}+2 \epsilon\left(\frac{\alpha_{3} \alpha_{4}-\alpha_{2} \alpha_{5}}{\alpha_{1} \alpha_{4}-\alpha_{5}}\right) y z+D_{3} z^{2}
$$

which are contained in (2.9) and by using the inequality $|y z| \leq \frac{1}{2}\left(y^{2}+z^{2}\right)$, we obtain

$$
v_{5} \geq D_{2} y^{2}+D_{3} z^{2}-\epsilon\left(\frac{\alpha_{3} \alpha_{4}-\alpha_{2} \alpha_{5}}{\alpha_{1} \alpha_{4}-\alpha_{5}}\right)\left(y^{2}+z^{2}\right) \geq D_{6}\left(y^{2}+z^{2}\right),
$$

for some $D_{6}>0, D_{6}=\frac{1}{2} \min \left\{D_{2}, D_{3}\right\}$, if

$$
\epsilon \leq \frac{\alpha_{1} \alpha_{4}-\alpha_{5}}{2\left(\alpha_{3} \alpha_{4}-\alpha_{2} \alpha_{5}\right)} \min \left\{D_{2}, D_{3}\right\}
$$

By using the previous inequality, we get from (2.9) that

$$
\begin{aligned}
2 V \geq & D_{1} H(x)+\left(D_{2}+D_{6}\right) y^{2}+\left(D_{3}+D_{6}\right) z^{2}+D_{4} w^{2}+D_{5} u^{2} \\
& +2 \lambda \int_{-r}^{0} \int_{t+s}^{t} w^{2}(\theta) \mathrm{d} \theta \mathrm{d} s+2 \rho \int_{-r}^{0} \int_{t+s}^{t} u^{2}(\theta) \mathrm{d} \theta \mathrm{d} s .
\end{aligned}
$$

As a result, since the integrals

$$
2 \lambda \int_{-r}^{0} \int_{t+s}^{t} w^{2}(\theta) \mathrm{d} \theta \mathrm{d} s \text { and } 2 \rho \int_{-r}^{0} \int_{t+s}^{t} u^{2}(\theta) \mathrm{d} \theta \mathrm{d} s
$$

are non-negative, it is obvious that there exists a positive constant $D_{7}$ which satisfies the following inequality

$$
V\left(x_{t}, y_{t}, z_{t}, w_{t}, u_{t}\right) \geq D_{7}\left\{H(x)+y^{2}+z^{2}+w^{2}+u^{2}\right\},
$$

where

$$
D_{7}=\frac{1}{2} \min \left\{D_{1}, D_{2}+D_{6}, D_{3}+D_{6}, D_{4}, D_{5}\right\} .
$$

Now by a direct calculation from (1.2) and (2.5) one finds 


$$
\begin{aligned}
& \frac{\mathrm{d} V}{\mathrm{~d} t}\left(x_{t}, y_{t}, z_{t}, w_{t}, u_{t}\right)=-\left[\psi(x(t-r), y(t-r), z(t-r), w(t-r), u(t-r))-\alpha_{1}\right] u^{2} \\
& -\left[\alpha_{1} \frac{f(w)}{w}-\left\{\alpha_{3}+\frac{\alpha_{1} \alpha_{4}\left(\alpha_{1} \alpha_{2}-\alpha_{3}\right)}{\alpha_{1} \alpha_{4}-\alpha_{5}}-\delta\right\}\right] w^{2} \\
& -\left[\frac{\alpha_{4}\left(\alpha_{1} \alpha_{2}-\alpha_{3}\right)}{\alpha_{1} \alpha_{4}-\alpha_{5}} \frac{\phi(z)}{z}-\left\{\delta \alpha_{2}+\left(\alpha_{1} g^{\prime}(y)-\alpha_{5}\right)\right\}\right] z^{2} \\
& -\left[\delta \frac{g(y)}{y}-\frac{\alpha_{4}\left(\alpha_{1} \alpha_{2}-\alpha_{3}\right)}{\alpha_{1} \alpha_{4}-\alpha_{5}} h^{\prime}(x)\right] y^{2}-\alpha_{1}\left[\psi(x, y, z, w, u)-\alpha_{1}\right] w u \\
& -\left[\frac{\phi(z)}{z}-\alpha_{3}\right] u z-\left[\alpha_{4}-g^{\prime}(y)\right] w z-\delta\left[\frac{\phi(z)}{z}-\alpha_{3}\right] y z \\
& -\alpha_{1}\left[\alpha_{5}-h^{\prime}(x)\right] y z-\frac{\alpha_{4}\left(\alpha_{1} \alpha_{2}-\alpha_{3}\right)}{\alpha_{1} \alpha_{4}-\alpha_{5}}\left[\psi(x, y, z, w, u)-\alpha_{1}\right] z u \\
& -\left[\alpha_{5}-h^{\prime}(x)\right] w y-\delta\left[\psi(x, y, z, w, u)-\alpha_{1}\right] y u \\
& -\delta\left[\frac{f(w)}{w}-\alpha_{2}\right] w y-\frac{\alpha_{4}\left(\alpha_{1} \alpha_{2}-\alpha_{3}\right)}{\alpha_{1} \alpha_{4}-\alpha_{5}}\left[\frac{f(w)}{w}-\alpha_{2}\right] w z \\
& +u \int_{t-r}^{t} f^{\prime}(w(s)) u(s) \mathrm{d} s+u \int_{t-r}^{t} \phi^{\prime}(z(s)) w(s) \mathrm{d} s \\
& +\alpha_{1} w \int_{t-r}^{t} f^{\prime}(w(s)) u(s) \mathrm{d} s+\alpha_{1} w \int_{t-r}^{t} \phi^{\prime}(z(s)) w(s) \mathrm{d} s \\
& +\frac{\alpha_{4}\left(\alpha_{1} \alpha_{2}-\alpha_{3}\right)}{\alpha_{1} \alpha_{4}-\alpha_{5}} z \int_{t-r}^{t} f^{\prime}(w(s)) u(s) \mathrm{d} s \\
& +\frac{\alpha_{4}\left(\alpha_{1} \alpha_{2}-\alpha_{3}\right)}{\alpha_{1} \alpha_{4}-\alpha_{5}} z \int_{t-r}^{t} \phi^{\prime}(z(s)) w(s) \mathrm{d} s \\
& +\delta y \int_{t-r}^{t} f^{\prime}(w(s)) u(s) \mathrm{d} s+\delta y \int_{t-r}^{t} \phi^{\prime}(z(s)) w(s) \mathrm{d} s \\
& +\rho u^{2} r-\rho \int_{t-r}^{t} u^{2}(s) \mathrm{d} s+\lambda w^{2} r-\lambda \int_{t-r}^{t} w^{2}(s) \mathrm{d} s .
\end{aligned}
$$

Making use of the assumptions ii)-vi), (2.3) and (2.6), we get

$$
\begin{aligned}
\psi(x(t-r), y(t-r), z(t-r), w(t-r), u(t-r))-\alpha_{1} \geq 2 \epsilon_{o}, \\
\alpha_{1} \frac{f(w)}{w}-\left\{\alpha_{3}+\frac{\alpha_{1} \alpha_{4}\left(\alpha_{1} \alpha_{2}-\alpha_{3}\right)}{\alpha_{1} \alpha_{4}-\alpha_{5}}-\delta\right\} \\
=\alpha_{1}\left[\frac{f(w)}{w}-\alpha_{2}\right]+\left\{\alpha_{1} \alpha_{2}-\alpha_{3}+\delta-\frac{\alpha_{1} \alpha_{4}\left(\alpha_{1} \alpha_{2}-\alpha_{3}\right)}{\alpha_{1} \alpha_{4}-\alpha_{5}}\right\} \geq \epsilon, \\
\quad \frac{\alpha_{4}\left(\alpha_{1} \alpha_{2}-\alpha_{3}\right)}{\alpha_{1} \alpha_{4}-\alpha_{5}} \frac{\phi(z)}{z}-\left\{\delta \alpha_{2}+\left(\alpha_{1} g^{\prime}(y)-\alpha_{5}\right)\right\} \\
\geq \frac{\left(\alpha_{4} \alpha_{3}-\alpha_{2} \alpha_{5}\right)\left(\alpha_{1} \alpha_{2}-\alpha_{3}\right)}{\alpha_{1} \alpha_{4}-\alpha_{5}}-\left\{\alpha_{1} g^{\prime}(y)-\alpha_{5}\right\}-\epsilon \alpha_{2} \\
\geq 2 \epsilon \alpha_{2}-\epsilon \alpha_{2}=\epsilon \alpha_{2},
\end{aligned}
$$

and

$$
\left[\delta \frac{g(y)}{y}-\frac{\alpha_{4}\left(\alpha_{1} \alpha_{2}-\alpha_{3}\right)}{\alpha_{1} \alpha_{4}-\alpha_{5}} h^{\prime}(x)\right] \geq \epsilon \alpha_{4}+\frac{\alpha_{4} \alpha_{5}\left(\alpha_{1} \alpha_{2}-\alpha_{3}\right)}{\alpha_{1} \alpha_{4}-\alpha_{5}}-\frac{\alpha_{4} \alpha_{5}\left(\alpha_{1} \alpha_{2}-\alpha_{3}\right)}{\alpha_{1} \alpha_{4}-\alpha_{5}}=\epsilon \alpha_{4},
$$


By v), vi) and (2.6).

By using the assumptions $\left|f^{\prime}(w(s))\right| \leq M$ and $\left|\phi^{\prime}(z(s))\right| \leq L$ of the theorem and inequality $2|a b| \leq a^{2}+b^{2}$, we obtain the following inequalities

$$
\begin{gathered}
u \int_{t-r}^{t} f^{\prime}(w(s)) u(s) \mathrm{d} s \leq \frac{M}{2} r u^{2}(t)+\frac{M}{2} \int_{t-r}^{t} u^{2}(s) \mathrm{d} s, \\
\alpha_{1} w \int_{t-r}^{t} f^{\prime}(w(s)) u(s) \mathrm{d} s \leq \frac{\alpha_{1} M}{2} r w^{2}(t)+\frac{\alpha_{1} M}{2} \int_{t-r}^{t} u^{2}(s) \mathrm{d} s, \\
\frac{\alpha_{4}\left(\alpha_{1} \alpha_{2}-\alpha_{3}\right)}{\alpha_{1} \alpha_{4}-\alpha_{5}} z \int_{t-r}^{t} f^{\prime}(w(s)) u(s) \mathrm{d} s \leq \frac{\alpha_{4}\left(\alpha_{1} \alpha_{2}-\alpha_{3}\right)}{\alpha_{1} \alpha_{4}-\alpha_{5}} \frac{M}{2} r z^{2}(t)+\frac{\alpha_{4}\left(\alpha_{1} \alpha_{2}-\alpha_{3}\right)}{\alpha_{1} \alpha_{4}-\alpha_{5}} \frac{M}{2} \int_{t-r}^{t} u^{2}(s) \mathrm{d} s, \\
\delta y \int_{t-r}^{t} f^{\prime}(w(s)) u(s) \mathrm{d} s \leq \frac{\delta M}{2} r y^{2}(t)+\frac{\delta M}{2} \int_{t-r}^{t} u^{2}(s) \mathrm{d} s, \\
u \int_{t-r}^{t} \phi^{\prime}(z(s)) w(s) \mathrm{d} s \leq \frac{L}{2} r u^{2}(t)+\frac{L}{2} \int_{t-r}^{t} w^{2}(s) \mathrm{d} s, \\
\alpha_{1} w \int_{t-r}^{t} \phi^{\prime}(z(s)) w(s) \mathrm{d} s \leq \frac{\alpha_{1} L}{2} r w^{2}(t)+\frac{\alpha_{1} L}{2} \int_{t-r}^{t} w^{2}(s) \mathrm{d} s, \\
\frac{\alpha_{4}\left(\alpha_{1} \alpha_{2}-\alpha_{3}\right)}{\alpha_{1} \alpha_{4}-\alpha_{5}} z \int_{t-r}^{t} \phi^{\prime}(z(s)) w(s) \mathrm{d} s \leq \frac{\alpha_{4}\left(\alpha_{1} \alpha_{2}-\alpha_{3}\right)}{\alpha_{1} \alpha_{4}-\alpha_{5}} \frac{L}{2} r z^{2}(t)+\frac{\alpha_{4}\left(\alpha_{1} \alpha_{2}-\alpha_{3}\right)}{\alpha_{1} \alpha_{4}-\alpha_{5}} \frac{L}{2} \int_{t-r}^{t} w^{2}(s) \mathrm{d} s,
\end{gathered}
$$

and

$$
\delta y \int_{t-r}^{t} \phi^{\prime}(z(s)) w(s) \mathrm{d} s \leq \frac{\delta L}{2} r y^{2}(t)+\frac{\delta L}{2} \int_{t-r}^{t} w^{2}(s) \mathrm{d} s .
$$

Replacing the last equality and the preceding inequalities into (2.12), we obtain

$$
\begin{aligned}
\frac{\mathrm{d} V}{\mathrm{~d} t} \leq & -\left[\frac{\epsilon \alpha_{4}}{6}-\frac{\delta(L+M)}{2} r\right] y^{2}-\left[\frac{\epsilon \alpha_{2}}{7}-\frac{\alpha_{4}(L+M)\left(\alpha_{1} \alpha_{2}-\alpha_{3}\right)}{2\left(\alpha_{1} \alpha_{4}-\alpha_{5}\right)} r\right] z^{2} \\
& -\left[\frac{\epsilon}{6}-\left\{\frac{\alpha_{1}(L+M)}{2}+\lambda\right\} r\right] \omega^{2}-\left[\epsilon_{o}-\left(\frac{L+M}{2}+\rho\right) r\right] u^{2} \\
& -\left[\lambda-\left\{\frac{L}{2}+\frac{\alpha_{1} L}{2}+\frac{\delta L}{2}+\frac{\alpha_{4}\left(\alpha_{1} \alpha_{2}-\alpha_{3}\right)}{2\left(\alpha_{1} \alpha_{4}-\alpha_{5}\right)} L\right\}\right] \int_{t-r}^{t} \omega^{2}(s) \mathrm{d} s \\
& -\left[\rho-\left\{\frac{M}{2}+\frac{\alpha_{1} M}{2}+\frac{\delta M}{2}+\frac{\alpha_{4}\left(\alpha_{1} \alpha_{2}-\alpha_{3}\right)}{2\left(\alpha_{1} \alpha_{4}-\alpha_{5}\right)} M\right\}\right] \int_{t-r}^{t} u^{2}(s) \mathrm{d} s-\sum_{k=6}^{15} v_{k},
\end{aligned}
$$

where

$$
\begin{aligned}
& v_{6}:=\frac{1}{8}\left(\psi-\alpha_{1}\right) u^{2}+\alpha_{1}\left(\psi-\alpha_{1}\right) w u+\frac{\epsilon}{6} w^{2}, \\
& v_{7}:=\frac{1}{8}\left(\psi-\alpha_{1}\right) u^{2}+\left[\frac{\phi(z)}{z}-\alpha_{3}\right] u z+\frac{\epsilon \alpha_{2}}{7} z^{2}, \\
& v_{8}:=\frac{\epsilon \alpha_{4}}{6} y^{2}+\delta\left[\frac{\phi(z)}{z}-\alpha_{3}\right] y z+\frac{\epsilon \alpha_{2}}{7} z^{2}, \\
& v_{9}:=\frac{1}{8}\left(\psi-\alpha_{1}\right) u^{2}+\frac{\alpha_{4}\left(\alpha_{1} \alpha_{2}-\alpha_{3}\right)}{\alpha_{1} \alpha_{4}-\alpha_{5}}\left(\psi-\alpha_{1}\right) u z+\frac{\epsilon \alpha_{2}}{7} z^{2}, \\
& v_{10}:=\frac{1}{8}\left(\psi-\alpha_{1}\right) u^{2}+\delta\left(\psi-\alpha_{1}\right) u y+\frac{\epsilon \alpha_{4}}{6} y^{2},
\end{aligned}
$$




$$
\begin{aligned}
& v_{11}:=\frac{\epsilon}{6} w^{2}+\frac{\alpha_{4}\left(\alpha_{1} \alpha_{2}-\alpha_{3}\right)}{\alpha_{1} \alpha_{4}-\alpha_{5}}\left[\frac{f(w)}{w}-\alpha_{2}\right] w z+\frac{\epsilon \alpha_{2}}{7} z^{2}, \\
& v_{12}:=\frac{\epsilon}{6} w^{2}+\delta\left[\frac{f(w)}{w}-\alpha_{2}\right] w y+\frac{\epsilon \alpha_{4}}{6} y^{2}, \\
& v_{13}:=\frac{\epsilon}{6} w^{2}+\left[\alpha_{4}-g^{\prime}(y)\right] w z+\frac{\epsilon \alpha_{2}}{7} z^{2}, \\
& v_{14}:=\frac{\epsilon \alpha_{4}}{6} y^{2}+\alpha_{1}\left[\alpha_{5}-h^{\prime}(x)\right] y z+\frac{\epsilon \alpha_{2}}{7} z^{2}
\end{aligned}
$$

and

$$
v_{15}:=\frac{\epsilon}{6} w^{2}+\left[\alpha_{5}-h^{\prime}(x)\right] w y+\frac{\epsilon \alpha_{4}}{6} y^{2} .
$$

It is clear that the expressions given by $v_{6}, \cdots, v_{14}$ and $v_{15}$ represent certain specific quadratic forms, respectively.

Making use of the basic information on the positive semi-definite of a quadratic form, one can easily conclude that $v_{6} \geq 0, v_{7} \geq 0, v_{8} \geq 0, v_{9} \geq 0, v_{10} \geq 0, v_{11} \geq 0, v_{12} \geq 0, v_{13} \geq 0, v_{14} \geq 0$ and $v_{15} \geq 0$ provided that

$$
\begin{gathered}
\left(\psi-\alpha_{1}\right) \leq \frac{\epsilon}{12 \alpha_{1}^{2}}, \quad\left(\psi-\alpha_{1}\right) \leq \frac{\epsilon \alpha_{2}\left(\alpha_{1} \alpha_{4}-\alpha_{5}\right)^{2}}{14 \alpha_{4}^{2}\left(\alpha_{1} \alpha_{2}-\alpha_{3}\right)^{2}}, \quad\left(\psi-\alpha_{1}\right) \leq \frac{\epsilon \alpha_{4}}{12 \delta^{2}}, \\
{\left[\frac{\phi(z)}{z}-\alpha_{3}\right]^{2} \leq \frac{2 \epsilon^{2} \alpha_{2} \alpha_{4}}{21 \delta^{2}}, \quad\left[\frac{\phi(z)}{z}-\alpha_{3}\right]^{2} \leq \frac{\epsilon \epsilon_{o} \alpha_{2}}{7},} \\
{\left[\frac{f(w)}{w}-\alpha_{2}\right]^{2} \leq \frac{2 \epsilon^{2} \alpha_{2}\left(\alpha_{1} \alpha_{4}-\alpha_{5}\right)^{2}}{21 \alpha_{4}^{2}\left(\alpha_{1} \alpha_{2}-\alpha_{3}\right)^{2}}, \quad\left[\frac{f(w)}{w}-\alpha_{2}\right]^{2} \leq \frac{\epsilon^{2} \alpha_{4}}{9 \delta^{2}},} \\
{\left[\alpha_{5}-h^{\prime}(x)\right]^{2} \leq \frac{2 \epsilon^{2} \alpha_{2} \alpha_{4}}{21 \alpha_{1}^{2}}, \quad\left[\alpha_{5}-h^{\prime}(x)\right]^{2} \leq \frac{\epsilon^{2} \alpha_{4}}{9}}
\end{gathered}
$$

and

$$
\left[\alpha_{4}-g^{\prime}(y)\right]^{2} \leq \frac{2 \epsilon^{2} \alpha_{2}}{21}
$$

respectively.

Thus in view of the above discussion and inequality (2.13), it follows that

$$
\begin{aligned}
\frac{\mathrm{d} V}{\mathrm{~d} t} \leq & -\left[\frac{\epsilon \alpha_{4}}{6}-\frac{\delta(L+M)}{2} r\right] y^{2}-\left[\frac{\epsilon \alpha_{2}}{7}-\frac{\alpha_{4}(L+M)\left(\alpha_{1} \alpha_{2}-\alpha_{3}\right)}{2\left(\alpha_{1} \alpha_{4}-\alpha_{5}\right)} r\right] z^{2} \\
& -\left[\frac{\epsilon}{6}-\left\{\frac{\alpha_{1}(L+M)}{2}+\lambda\right\} r\right] w^{2}-\left[\epsilon_{o}-\left(\frac{L+M}{2}+\rho\right) r\right] u^{2} \\
& -\left[\lambda-\left\{\frac{L}{2}+\frac{\alpha_{1} L}{2}+\frac{\delta L}{2}+\frac{\alpha_{4}\left(\alpha_{1} \alpha_{2}-\alpha_{3}\right)}{2\left(\alpha_{1} \alpha_{4}-\alpha_{5}\right)} L\right\}\right] \int_{t-r}^{t} w^{2}(s) \mathrm{d} s \\
& -\left[\rho-\left\{\frac{M}{2}+\frac{\alpha_{1} M}{2}+\frac{\delta M}{2}+\frac{\alpha_{4}\left(\alpha_{1} \alpha_{2}-\alpha_{3}\right)}{2\left(\alpha_{1} \alpha_{4}-\alpha_{5}\right)} M\right\}\right] \int_{t-r}^{t} u^{2}(s) \mathrm{d} s .
\end{aligned}
$$

So we can choose the constants $\lambda$ and $\rho$ as the following 


$$
\lambda=\frac{L}{2}+\frac{\alpha_{1} L}{2}+\frac{\delta L}{2}+\frac{\alpha_{4}\left(\alpha_{1} \alpha_{2}-\alpha_{3}\right)}{2\left(\alpha_{1} \alpha_{4}-\alpha_{5}\right)} L
$$

and

$$
\rho=\frac{M}{2}+\frac{\alpha_{1} M}{2}+\frac{\delta M}{2}+\frac{\alpha_{4}\left(\alpha_{1} \alpha_{2}-\alpha_{3}\right)}{2\left(\alpha_{1} \alpha_{4}-\alpha_{5}\right)} M,
$$

then the inequality in (2.14) implies that

$$
\begin{aligned}
\frac{\mathrm{d} V}{\mathrm{~d} t} \leq & -\left[\frac{\epsilon \alpha_{4}}{6}-\frac{\delta(L+M)}{2} r\right] y^{2}-\left[\frac{\epsilon \alpha_{2}}{7}-\frac{\alpha_{4}(L+M)\left(\alpha_{1} \alpha_{2}-\alpha_{3}\right)}{2\left(\alpha_{1} \alpha_{4}-\alpha_{5}\right)} r\right] z^{2} \\
& -\left[\frac{\epsilon}{6}-\left\{\frac{\alpha_{1}(L+M)}{2}+\lambda\right\} r\right] w^{2}-\left[\epsilon_{o}-\left(\frac{L+M}{2}+\rho\right) r\right] u^{2} .
\end{aligned}
$$

Hence one can easily get from (2.15) that

$$
\frac{\mathrm{d} V}{\mathrm{~d} t}\left(x_{t}, y_{t}, z_{t}, w_{t}, u_{t}\right) \leq-D_{8} y^{2}-D_{9} z^{2}-D_{10} w^{2}-D_{11} u^{2} \leq 0,
$$

for some positive constants $D_{i},(i=8, \cdots, 11)$, provided that

$$
r<\min \left\{\frac{\epsilon \alpha_{4}}{6 \delta(L+M)}, \frac{\epsilon \alpha_{2}\left(\alpha_{1} \alpha_{4}-\alpha_{5}\right)}{7 \alpha_{4}(L+M)\left(\alpha_{1} \alpha_{2}-\alpha_{3}\right)}, \frac{\epsilon}{6 \alpha_{1}(L+M)+12 \lambda}, \frac{\epsilon_{o}}{L+M+2 \rho}\right\} .
$$

Finally, it follows that $\frac{\mathrm{d} V}{\mathrm{~d} t}=0$ if and only if $x_{t}=y_{t}=z_{t}=w_{t}=u_{t}=0, \frac{\mathrm{d} V(\varphi)}{\mathrm{d} t}<0$ for $\varphi \neq 0$ and $V(\varphi) \geq u(|\varphi(0)|) \geq 0$.

Thus all the conditions of Theorem 2.2 are satisfied. This shows that the zero solution of (1.1) is globally asymptotically stable.

Then the proof of Theorem 2.3 is completed.

\section{References}

[1] Adesina, O.A. (2007) Convergence Results for Solutions of a Certain Fifth-Order Nonlinear Differential Equation. Nonlinear Studies, 14, 289-300.

[2] Bereketoglu, H. (1998) Asymptotic Stability of a Fourth-Order Delay Differential Equation. Dynamic Systems an Applications, 7, 105-115.

[3] Burton, T.A. (1983) Volterra Integral and Differential Equations. Academic Press, New York.

[4] Burton, T.A. (1985) Stability and Periodic Solutions of Ordinary and Functional Differential Equations. Academic Press, New York.

[5] Chukwu, E.N. (1978) On the Boundedness and the Existence of a Periodic Solution of Some Nonlinear Third-Order Delay Differential Equation. Atti Accad. Naz. Lincei Rend. Cl. Sci. Fis. Mat. Nat., 64, 440-447.

[6] Harrow, M. (1967) A Stability Result for Solutions of Certain Fourth-Order Homogeneous Differential Equations. Journal London Mathematical Society, 42, 51-56. http://dx.doi.org/10.1112/jlms/s1-42.1.51

[7] Harrow, M. (1970) On the Boundedness and the Stability of Solutions of Some Differential Equations of the FourthOrder. SIAM Journal on Mathematical Analysis, 1, 27-32. http://dx.doi.org/10.1137/0501002

[8] Hale, J.K. (1977) Theory of Functional Differential Equations. Springer-Verlag, New York. http://dx.doi.org/10.1007/978-1-4612-9892-2

[9] Kolmanovskii, V. and Myshkis, A. (1999) Introduction to the Theory and Applications of Functional Differential Equations. Kluwer Academic Publishers, Dordrecht. http://dx.doi.org/10.1007/978-94-017-1965-0

[10] Makay, G. (1994) On the Asymptotic Stability of the Solutions of Functional Differential Equations with Infinite Delay. Journal of Differential Equations, 108, 139-151. http://dx.doi.org/10.1006/jdeq.1994.1029

[11] Okoronkwo, E.O. (1989) On Stability and Boundedness of Solutions of a Certain Fourth-Order Delay Differential Eq- 
uation. International Journal of Mathematics and Mathematical Sciences, 12, 589-602. http://dx.doi.org/10.1155/S0161171289000724

[12] Reissing, R., Sansone, E. and Conti, R. (1974) Non-Linear Differential Equations of Higher-Order. Noordhoof, Toom.

[13] Sadek, A.I. (2003) Stability and Boundedness of a Kind of Third-Order Delay Differential System. Applied Mathematics Letters, 16, 657-662. http://dx.doi.org/10.1016/S0893-9659(03)00063-6

[14] Sadek, A.I. (2004) On the Stability of Solutions of Certain Fourth-Order Delay Differential Equations. Applied Mathematics and Computation, 148, 587-597. http://dx.doi.org/10.1016/S0096-3003(02)00925-6

[15] Sadek, A.I. (2005) On the Stability of Solutions of Some Non-Autonomous Delay Differential Equations of the ThirdOrder. Asymptotic Analysis, 43, 1-7.

[16] Li, S.L. and Wen, L.Z. (1987) Functional Differential Equations. Hunan Science and Technology Press, Changsha.

[17] Sinha, A.S.C. (1973) On Stability of Solutions of Some Third and Fourth-Order Delay Differential Equations. Information and Control, 23, 165-172. http://dx.doi.org/10.1016/S0019-9958(73)90651-7

[18] Tejumola, H.O. and Tchegnani, B. (2000) Stability, Boundedness and Existence of Periodic Solutions of Some Third and Fourth-Order Nonlinear Delay Differential Equations. Journal of the Nigerian Mathematics Society, 19, 9-19.

[19] Tunç, C. (2002) A Study of the Stability and Boundedness of the Solutions of Nonlinear Differential Equations of the Fifth-Order. Indian Journal of Pure and Applied Mathematics, 33, 519-529.

[20] Tunç, C. (2005) Some Stability Results for the Solutions of Certain Fourth-Order Delay Differential Equations. Differential Equations \& Applications, 4, 165-174.

[21] Tunç, C. (2006) On the Stability of Solutions of Certain Fourth-Order Delay Differential Equations. Applied Mathematics and Mechanics, 27, 1141-1148.

[22] Tunç, C. (2008) On the Stability of Solutions to a Certain Fourth-Order Delay Differential Equation. Nonlinear Dynamics, 51, 71-81. http://dx.doi.org/10.1007/s11071-006-9192-z

[23] Tunç, C. (2010) On Asymptotic Stability of Solutions of Fifth-Order Nonlinear Differential Equations with Delay. Functional Differential Equations, 17, 355-370.

[24] Tunç, C. (2013) New Results on the Stability and Boundedness of Nonlinear Differential Equations of Fifth-Order with Multiple Deviating Arguments. Bulletin of the Malaysian Mathematical Sciences Society, 36, 671-682.

[25] Yoshizawa, T. (1966) Stability Theory by Lyapunov’s Second Method. The Mathematical Society of Japan.

[26] Qin, Y., Liu, Y. and Wang, L. (1966) Stability of Motion for Dynamic Systems with Delay. Academic Press, Beijing.

[27] Zhu, Y. (1992) On Stability, Boundedness and Existence of Periodic Solution of a Kind of Third-Order Nonlinear Delay Differential System. Annals of Differential Equations, 8, 249-259.

[28] Lyapunov, A.M. (1966) Stability of Motion. Academic Press, London.

[29] Tunç, C. (2010) On the Stability of Solutions of Nonlinear Differential Equations of Fifth-Order with Delay. Mathematical Communications, 15, 261-272.

[30] Abou-El-Ela, A.M.A., Sadek, A.I. and Mahmoud, A.M. (2011) On the Stability of Solutions of a Certain Nonlinear Delay Differential Equation of the Fifth-Order. Proceedings of the 3rd Scientific Conference for Young Researchers, Assiut, 19-20 April 2011.

[31] Burton, T.A. and Hering, G. (1994) Lyapunov Theory for Functional Differential Equations. Rocky Mountain Journal of Mathematics, 24, 3-17. http://dx.doi.org/10.1216/rmjm/1181072449

[32] Hale, J.K. (1965) Sufficient Conditions for Stability and Instability of Autonomous Functional-Differential Equations. Journal of Differential Equations, 1, 452-482. http://dx.doi.org/10.1016/0022-0396(65)90005-7 
Scientific Research Publishing (SCIRP) is one of the largest Open Access journal publishers. It is currently publishing more than 200 open access, online, peer-reviewed journals covering a wide range of academic disciplines. SCIRP serves the worldwide academic communities and contributes to the progress and application of science with its publication.

Other selected journals from SCIRP are listed as below. Submit your manuscript to us via either submit@scirp.org or Online Submission Portal.
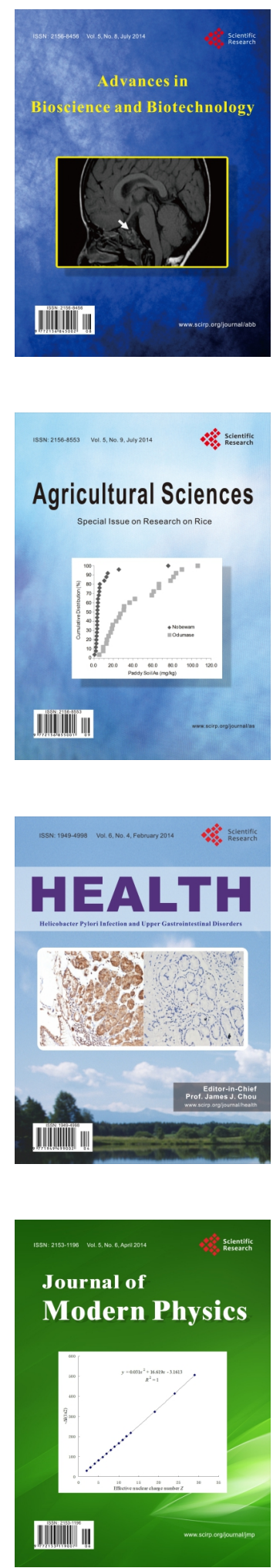
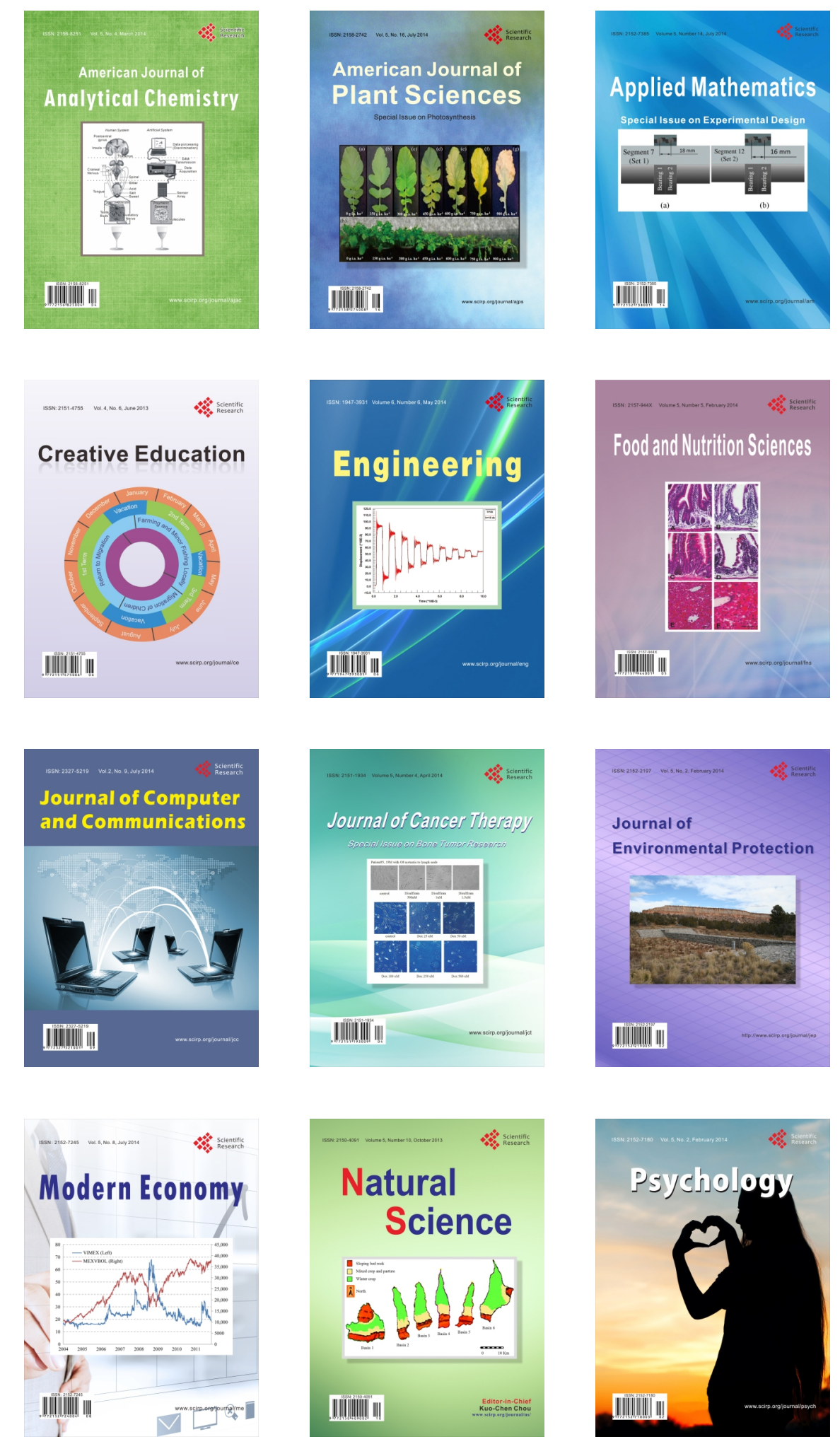